\title{
AUTOMATIC PATIENT CONTROLLED ANALGESIA INJECTOR
}

\author{
A.Srilatha Reddy ${ }^{1}$, R. Venkata Raghava ${ }^{2}$, Mariya Fatima ${ }^{3}$, S. Richardson ${ }^{4}$ \\ ${ }^{I}$ Assistant Professor, Dept of BME, B.V.Raju Inst. of Tech., Narsapur, Medak, T. S., India \\ ${ }^{2}$ He is pursuing Bachelor's degree, BME Dept B. V. Raju Inst. of Tech., Narsapur, Medak, T. S., India \\ ${ }^{3}$ She is pursuing Bachelor's degree, BME Dept B. V. Raju Inst. of Tech., Narsapur, Medak, T. S, India \\ ${ }^{4}$ He is pursuing Bachelor's degree, BME Dept B. V. Raju Inst. of Tech., Narsapur, Medak, T. S., India
}

\begin{abstract}
Patient-Controlled Analgesia (PCA) has become accepted as an important means of self-regulated relief from post-surgical pain. In commonly used PCA systems, patients use a hand-held push-button to indicate the presence of pain and initiate a predetermined bolus of drug infusion. A disadvantage of this system is that no means is provided to accommodate variations in the intensity of pain or the sensitivity of the patient to the analgesic in use apart from the frequency of button pushing. A fixed rate background infusion is usually an option.
\end{abstract}

A new adaptive PCA system is proposed to provide improved PCA through the use a variable background infusion, the provision for an extended high range of analgesic dosages and a novel handset which allows patients to rate their pain. The total system is under the control of an expert algorithm and is proposed to overcome some of the shortcomings of current systems.

The specially designed handset allows patients to indicate a range of pain intensities and so vary the level of drug administration. Data derived from the handset signals provide a basis for the expert system to adapt the drug dosage to patient sensitivity as well as pain intensity. The variable background infusion is used to supplement analgesic requested by patients and is periodically adjusted by the expert algorithm. In addition an Oximeter provides direct monitoring of the patient and this safety measure allows for a wider range of adaptation under expert system control.

Although clear superior pain relief from the adaptive system could not be statistically established for the small trial population, clinical trials on 20 patients at the Royal Melbourne Hospital have indicated that the system provides effective pain control and is well accepted by both patients and clinical staff.

Keywords: Patient controlled Analgesia, Analgesia, Bolus, opioids, Human-machine interface.

\section{INTRODUCTION}

Despite the existence of potent analgesics, many patients continue to experience inadequate pain Effective treatment of severe pain has been one of medicine's great challenges relief. This thesis addresses a way of improving postoperative pain relief by improving the method by which analgesics are administered. Patient-Controller Analgesia (PCA) is such a method [1].

\subsection{A General Description of PCA}

In its simplest form, a PCA system consists of an electronically-controlled infusion pump connected to a programmable device. When patients experience pain they trigger the infusion pump by means of a button or handset extending from the pump, causing it to deliver a preset dose of analgesic referred to as a 'bolus amount.' Following the infusion of the bolus, the timing device precludes the further administration of analgesic for a minimum time period usually called the 'lockout period'. The lockout period prevents a subsequent dose of analgesic being administered until the first dose has had time to take effect. Figure 1.1 below shows a simplified PCA system. - $* * *$

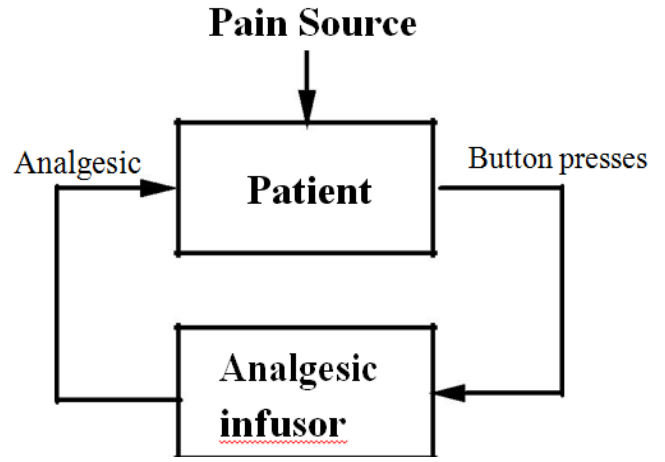

Fig 1.1 A general PCA system.
Modern PCA devices elaborate on the basic scheme outlined above by giving the patient and clinician greater control over the bolus amounts, lockout period, dosage limits and alarm conditions. Some devices also permit a low level background infusion to tide the patient over periods of sleep when no bolus requests are made.

The analgesic dosage levels at which patients experience pain relief vary greatly, as does the patient's sensitivity to pain. Using PCA, patients are able to determine their own analgesic requirements. 
The PCA devices discussed in this thesis are intended for post-operative patients unless stated otherwise. In a typical PCA application, patients judged as being suitable will receive pre-operative education in the use of PCA. Postoperatively, the patients again receive basic PCA education. Standard nursing care is carried out as for non-PCA patients[1].

\subsection{Rationale for this PCA System}

This project aims to explore the better methods of adapting intravenous PCA more closely to individual patient requirements by taking into account some of the psychological and physiological bases of pain relief as it relates to PCA. It is envisaged that the use of an expert knowledge base coupled to more sophisticated technology and improved user interfaces will form a vital step in providing improved pain relief. The system presented here will initially restrict itself to morphine as the analgesic agent although equianalgesic doses of pethidine, fentanyl or alfentanil are possible as well.

\subsubsection{Limitations and Areas of Possible}

\section{Improvements of Conventional PCA Systems.}

Conventional PCA is defined here as PCA using fixed bolus sizes and an optional fixed level background infusion as prescribed by the pain service in a hospital. Conventional PCA suffers from a number of shortcomings which are listed below in abbreviated form. Each of the points is more fully discussed and referenced in later chapters. The new system proposed in this thesis aims to address these shortcomings.

1) Difficulty in setting the appropriate bolus size given the large variations in analgesic amounts required for adequate analgesia between patients. The bolus size usually prescribed for the majority of patients does not vary very much. With conventional fixed bolus systems, the patient has only one degree of freedom, i.e. a bolus at a fixed level or no bolus. In current practice, this means that the only way in which a patient can access increasing amounts of analgesic for severe pain is through an increased number of bolus requests. However, as each request entails a minimum lockout period, higher dosages can be achieved only over some time. Furthermore, it is not possible to request less than the fixed bolus amount.

\subsection{Summary}

The overriding aim of this PCA system is to provide better pain relief in areas where conventional PCA technology is not at its optimum.

It is proposed to explore some of the theoretical underpinnings of PCA and to propose a PCA system which incorporates new hypotheses and propositions regarding self-administered pain relief. It is thought that such a system will be more adaptive to individual patient's need and will be able to provide improved analgesia.

\section{THE CASE FOR PCA}

PCA has gradually become a more popular pain management modality both with patients and clinical staff.

One of the major reasons for inadequate analgesia is the method of analgesic administration, in particular the practice of pro re nata (p.r.n.) and scheduled intramuscular (IM) injections at three or four hourly intervals[2]. This practice results in a peak and trough effect as illustrated in figure 2.1 below. . The patient experiences very large swings in blood concentration over the four hourly bolus intervals, with attendant large changes in analgesia. The range in which analgesia is experienced is relatively small, and as the plasma concentration varies the patient receives satisfactory analgesia only for a relatively short time.

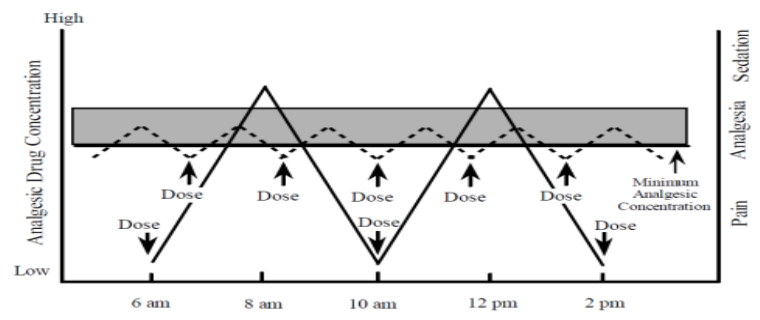

Fig 2.1 The peak and trough effects of conventional IM analgesia (solid line) compared to PCA (dashed line).

The problems in pain relief are discussed below and can best be subdivided into four groups: pharmacology, method of administration, the nature of pain itself, and patient variation.

\subsubsection{Opioids Used for Pain Management and Their}

\section{Unwanted Effects}

Pharmacologically, the ideal analgesic would have quick onset of action, no unwanted effects, no ceiling effects and no tolerance. No drug meets all these requirements, but morphine and pethidine (meperidine) present the best compromise and are the most frequently used[3].

\subsubsection{The Relationship between Plasma}

\section{Concentrations of Opioids and Analgesia}

The relationship between plasma concentration of opioids and analgesia is highly nonlinear. There is a point in many patients where a minimal increase in analgesic will take the patient from quite severe pain to near total analgesia. Despite increasing analgesic plasma concentrations, pain can be quite severe up to a "maximum concentration with severe pain' called the MCP point. Then with a minimal increase of opioid, the patient can be taken to almost complete analgesia at a level called the minimum effective analgesic concentration (MEAC)[3]. The concept of MEAC is not universally accepted.

\subsubsection{Patient Variation}

The method of administering opioid analgesics intramuscularly (IM) suffers from a number of problems which are largely exacerbated by inter patient variation. 
These are as follows:

1) Erratic inter patient variability in blood absorption time of the injected analgesic. Peak absorption times between 10 to 100 minutes.

2) The peak plasma concentrations of analgesics vary between patients for equal doses injected. In one study, using pethidine, the peak plasma concentration varied up to five-fold and the time to reach this peak varied seven-fold.

The age of the patient is a further source of variation in sensitivity to opioids. Older patients were found to be more sensitive than younger patients.

\subsubsection{An Evaluation of Traditional Methods of Analgesic Administration}

Traditional pain relief usually consists of prescribed bolus amounts injected intramuscularly as required or scheduled at regular intervals or of a continuous intravenous (IV) infusion. The prescribed amounts are loosely based on body weight and age[4]. This method does not optimize the usage of current analgesic drugs for several reasons .

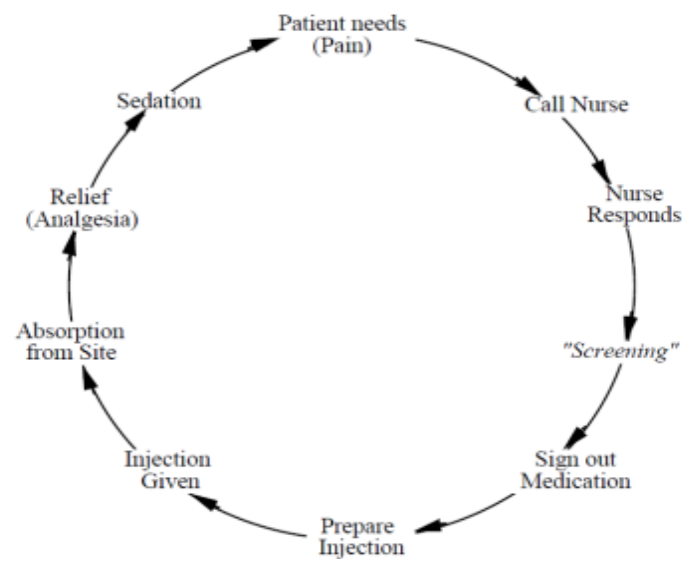

\subsection{Conclusion}

PCA addresses the problems of conventional p.r.n. pain relief in the following ways. By infusing a succession of small amounts of analgesic at the patient's request, the peak and trough effect commonly experienced with regular injections can be substantially reduced[5].

When making comparisons between PCA and regular scheduled injections, it is important to remember, that with 'attentive nursing care conventional intramuscular analgesia can become "on demand" and may be as effective as PCA'. In fact early trials of the PCA concept involved a nurseobserver to provide on-demand analgesia which effectively amounted to PCA.

\section{LITERATURE REVIEW}

PCA has developed its own specialist literature over the years. The aim of this chapter is to give an overview of that literature. Within the literature, a number of interest groups have focused on different aspects of PCA, such as evaluating PCA with respect to other methods of administering analgesia, using PCA for pain measurement or analgesic drug evaluation, and looking at the parameters of PCA systems themselves. This chapter identifies the major groups in the PCA literature and the dominant issues within each group.

\subsection{Classes of PCA}

Most PCA devices developed to date can be classified into the following four main groups

1) Bolus demand: The size and maximum dose are fixed.

2) Infusion demand: The demand varies a continuous variable infusion.

3) Bolus demand and constant infusion: The constant background infusion aims to provide a minimum level of analgesia and thus avoids the troughs in plasma drug levels when the patient is asleep.

4) Bolus demand and variable infusion: The system monitors the frequency of bolus demands and adjusts the background infusion accordingly.

Most commercial PCA systems belong to groups one and three[16].

\subsection{Current Issues in PCA}

There are a number of issues currently discussed in the PCA literature. Some of these issues have been identified and treated in the sections below.

\subsubsection{Minimum Effective Analgesic Concentration (MEAC)}

The concept of minimum effective analgesic concentration (MEAC) which was already alluded to in section II.1.2, has been the subject of lengthy debate in the PCA literature[13].

\subsubsection{Variations in MEAC and Natural Endorphins in the Cerebrospinal Fluid}

In an attempt to explain the large variations in the level of analgesic at which patients experience adequate analgesia, Tamsen has put forward the theory that an individual's analgesic plasma levels are indicative of his or her sensitivity to opiates and that this is in turn linked to an individual's endorphin activity in the brain. The study by Tamsen showed a significant inverse relationship between individual pre-operative cerebrospinal fluid (CSF) endorphin concentrations and meperidine (pethidine) plasma concentrations using PCA.

\subsubsection{Opioid Sparing Effects of PCA}

Some researchers have reported that the total amount of opioids used in PCA was lower than that used in conventional pro re nata (p.r.n.) administration while also giving more satisfactory analgesia[7]. 
A study by Zacharias found a lower total dose of morphine consumption using PCA when compared to constant infusion. It was pointed out in the study itself that this could be explained by the small bolus size $(0.5 \mathrm{mg})$ on the PCA and the longer than usual lockout time of $10 \mathrm{~min}$, such that the patients became tired of pressing the bolus request button repeatedly.

\section{THEORY OF THE ADAPTIVE PCA SYSTEM}

This chapter details the proposed PCA system and provides the theoretical framework for that proposal.

The proposed PCA system is intended to provide superior pain relief to that obtained with conventional bolus only or bolus plus fixed infusion systems. Four approaches make up the new system, these are:

1. Variable bolus administration and a variable selfadjusting background infusion.

2. A high adaptation feature for safely administering unusually high amounts of analgesic.

3. A specially designed handset allowing the patient to register varying levels of pain intensity and to receive appropriate analgesic amounts.

4. An expert system knowledge base embedded within the control algorithms of the system.

\subsection{A Theoretical Framework for the Patient}

\section{Handset and varying bolus amounts}

An effective handset rests on a number of theoretical foundations drawn from the areas of psychology, pharmacology and human-machine interface design[1].

\subsubsection{Psychological Aspects of the Patient Handset}

PCA is not only a purely physiological method of drug delivery but also has psychological aspects. The transition from the patient as mainly a recipient of medical treatment to the patient as a more active participant in medical treatment represents a fundamental change in approach with very important psychological implications[7].

\subsubsection{Human-Machine Interface Aspects of the}

\section{Patient Handset}

The design of the actual handset is based on general ergonomic guidelines of human-machine interface and tackles the problem of interfacing the PCA system in such a way that the patient can most easily communicate his or her level of pain intensity to the PCA system[9].

In the design of the handset the following guidelines were considered:

The method of indicating pain should be simple and easy to comprehend.

Commonly used conceptual icons and cues should be used as much as possible. This implies that the handset should build on currently accepted and familiar concepts whenever practical. Indicating pain and requesting a bolus should take a minimum of time[9]. The handset should be ergonomically well designed and user-friendly.

The handset should be safe from accidental triggering while still meeting the need for simplicity and ease of use.

In summary, the guidelines have been formulated to produce a handset which helps the patient externalize his or her subjective pain experience as accurately, quickly and easily as possible in order to obtain relief through the PCA system. In engineering terms, it is desired to have optimal coupling between the patient and the computer controlled pump. In software engineering terms the issue is one of 'userfriendly' interfacing.

\section{SYSTEM DESIGN}

The PCA system has been designed with the following practical guidelines:

1. Safety to patients: All reasonable care should be taken to eliminate as far as possible the danger of malfunction and injury.

2. Robustness. The system must be able to operate successfully and reliably in the demanding clinical environment. It should also record and store data for later analysis.

3. Critical conditions of the patient due to analgesia or other factors must be reported promptly and swiftly so that appropriate corrective action can be taken.

The PCA system described here uses a handset which is used for controlling the anesthesia machine automatically, depending upon the various biomedical parameters such as body temperature, heart rate, respiration rate. A block diagram of the complete system is shown below.

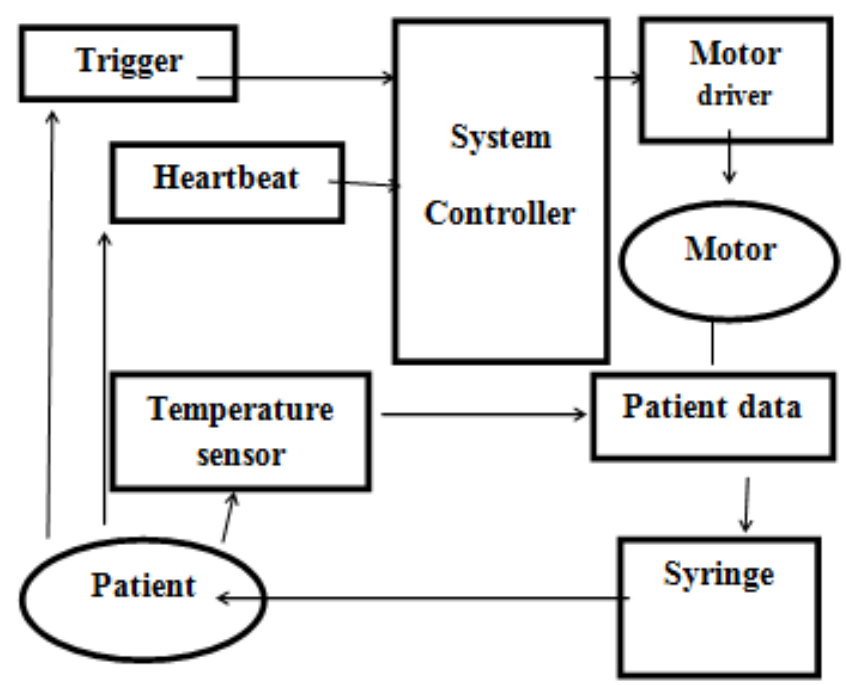

Fig 5.1 Block diagram of the PCA system

\subsection{PCA as a Feedback System}

The PCA system can be regarded as a feedback control loop in which the controlled variable is the level of pain and the feedback signal is the number of bolus requests. The 
patient's pain sensory system as a whole is the feedback signal generator, the output of which is a series of button presses to the controller controlling the syringe.

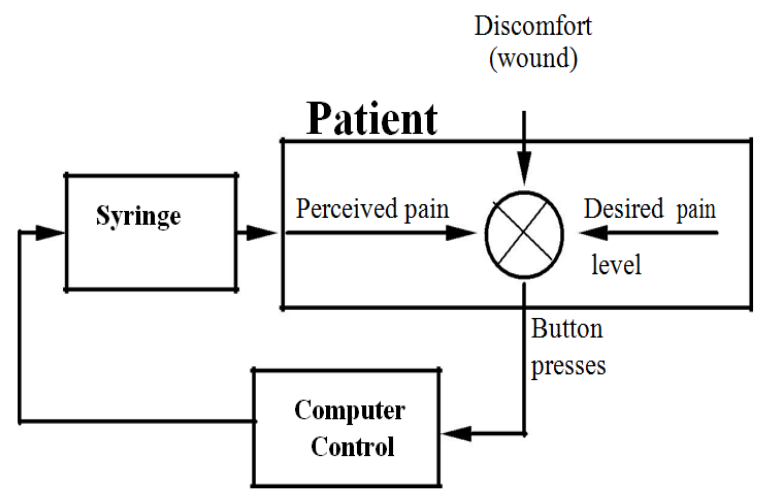

Fig 5.2 PCA as a feedback control system

\subsection{PCA System Hardware}

It consists of

1. LCD (16X2)

2. Stepper motor

3. Push button

4. $\quad$ Power suppy (A.c ilp i.e. 230V)

5. Transformer(step down 12V)

6. Rectifier

7. Filter(capacitive filter)

8. Voltage regulator (7805 and 7812)

\subsection{Introduction to L293d}

1. 600mA Output Current Capability Per Channel

2. 1.2A Peak Output Current per Channel

3. Enable Facility

4. Over temperature Protection

5. Logical "0" Input Voltage Up To $1.5 \mathrm{~V}$

6. Internal Clamp Diodes

\subsection{ATMEGA8}

The Atmel AVR core combines a rich instruction set with 32 general purpose working registers. All the 32 registers are directly connected to the Arithmetic Logic Unit (ALU), allowing two independent registers to be accessed in one single instruction executed in one clock cycle.

\subsubsection{Key features}

- High-performance, Low-power Atmel AVR 8-bit Microcontroller.

- $\quad$ Advanced RISC Architecture

- 130 Powerful Instructions - Most Single-clock Cycle Execution.

- $\quad 32 \times 8$ General Purpose Working Registers.

- High Endurance Non-volatile Memory segments.

- Data retention: 20 years at $85^{\circ} \mathrm{C} / 100$ years at $25^{\circ} \mathrm{C}$.

- $\quad$ Power Consumption at $4 \mathrm{Mhz}, 3 \mathrm{~V}, 25^{\circ} \mathrm{C}$

\subsection{Firmware Implementation of the Project Design}

The firmware programmed in ATMEGA8 is designed to communicate with DTMF and operates according to the digital output from DTMF. Therefore, the main firmware programmed can be divided into three parts:

1. Receive the Data from DTMF.

2. Analyzing the DTMF data

3. Switching ON/OFF A.C devices.

AVR STUDIO Is Used for the Automatic plant irrigation.

\section{PCA SAFETY}

The topic of safety in PCA and the steps needed to ensure patient safety at all times.

\subsection{The Dangers of PCA}

The main risk in PCA is posed by the fact that the opioid analgesics used will cause respiratory depression if given in sufficiently large doses. To prevent deliberate or accidental overdosing, a number of safety and risk-reducing features have been incorporated in the design of this particular PCA system.

\subsection{Inherent Safety}

Inherent safety has here been extrapolated to include the time decaying background infusion of adaptive PCA.

\subsection{Software Safety}

The underlying philosophy for software safety used in the adaptive PCA program is that 'every process must be within its legal range at every step'. If the slightest doubt concerning program correctness exists, the program is terminated in an orderly fashion.

\section{CONCLUSIION AND FUTURE SCOPE}

PCA constitutes an effective closed loop system, if one considers the patient as part of the control loop. PCA rests on the fundamental tenet that patients are best qualified to judge their level of pain and that the technology of PCA allows patients to assume control of their own pain relief. The proposed adaptive PCA system described here is a further step in this technology.

\section{REFERENCES}

[1]. Heiko E. R. Rudolph, “An Adaptive System for PatientControlled Analgesia", Vol 1, November 1995.

[2]. Bedder M. D., Soifer B. E., Mulhall J. J., "A comparison of patient-controlled analgesia and bolus PRN intravenous morphine in the intensive care environment", Clinical Journal of Pain, Vol 7, No 3, pp205-8, Sep 1991.

[3]. Bennett R. L., "Patient-controlled Analgesia for the Treatment of the Pain of Terminal Cancer: Description of an Initial Clinical Experience", in Harmer M.,Rosen M.,Vickers M. D., "Patient-controlled Analgesia", Blackwell Scientific Publ., 1985. 
[4]. Connor S. B., Quill T. J., Jacobs J. R., "Accuracy of Drug Infusion Pumps Under Computer Control”, IEEE Transactions on Biomedical Engineering, Vol 39 No 9 pp980-982, 1992.

[5]. Ferrante M. F.,Covino B. G., "Patient-Controlled Analgesia: A Historical Perspective", in: Ferrante M. F.,Ostheimer G. W., Covino B. G. eds.,'Patient-Controlled Analgesia”, Blackwell Scientific Publ., 1990.

[6]. Health Devices (Journal), "Patient-Controlled Analgesic Infusion Pumps", ECRL, Vol 17 No5 pp137-167, May 1988.

[7]. Jamieson R. N., Taft K., O'Hara J., Ferrante F. M., "Psychosocial and Pharmacolgic Predictors of Statisfaction with Intravenous Patient-Controlled Analgesia", Anesth Analg., Vol 77, pp121-125, 1993.

\section{Books Referred}

[8]. "An Adaptive System for Patient Controlled Analgesia" by Heiko E. R. Rudolph.

[9] "Analgesia" by Christoph Stein.

\section{BIOGRAPHIES}

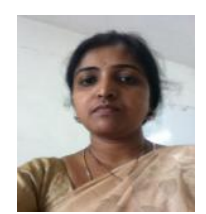

A.Srilatha Reddy, Assistant Professor in B.V.R.I.T since 10 years, M.Tech in Industrial Engineering, B.Tech in Biomedical Engineering

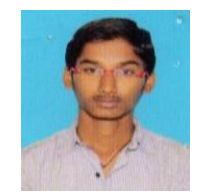

R.Venkat Raghava, Pursuing B.Tech 4th year in Biomedical engineering in B.V.R.I.T

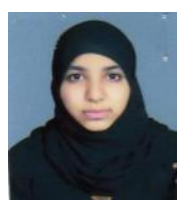

Mariya Fatima, Pursuing B.Tech $4^{\text {th }}$ year in Biomedical engineering in B.V.R.I.T

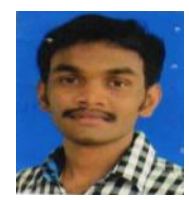

S.Richardson, Pursuing B.Tech $4^{\text {th }}$ year in Biomedical engineering in B.V.R.I.T 